Western University

Scholarship@Western

$2-25-2020$

\title{
Pulmonary Imaging Phenotypes of Chronic Obstructive Pulmonary Disease Using Multiparametric Response Maps
}

Jonathan L MacNeil

Dante P I Capaldi

Andrew R Westcott

Rachel L Eddy

Andrea L Barker

See next page for additional authors

Follow this and additional works at: https://ir.lib.uwo.ca/biophysicspub

Part of the Medical Biophysics Commons

Citation of this paper:

MacNeil, Jonathan L; Capaldi, Dante P I; Westcott, Andrew R; Eddy, Rachel L; Barker, Andrea L; McCormack, David G; Kirby, Miranda; and Parraga, Grace, "Pulmonary Imaging Phenotypes of Chronic Obstructive Pulmonary Disease Using Multiparametric Response Maps" (2020). Medical Biophysics Publications. 137.

https://ir.lib.uwo.ca/biophysicspub/137 


\section{Authors}

Jonathan L MacNeil, Dante P I Capaldi, Andrew R Westcott, Rachel L Eddy, Andrea L Barker, David G McCormack, Miranda Kirby, and Grace Parraga 


\title{
Pulmonary Imaging Phenotypes of Chronic Obstructive Pulmonary Disease Using Multiparametric Response Maps
}

\author{
Jonathan L. MacNeil, BSc • Dante P. I. Capaldi, PhD • Andrew R. Westcott, MSc ・ Rachel L. Eddy, BEng • \\ Andrea L. Barker, BSc • David G. McCormack, MD, FRCPC ・ Miranda Kirby, PhD • Grace Parraga, PhD
}

\begin{abstract}
From the Robarts Research Institute (J.L.M., A.R.W., R.L.E., A.L.B., G.P.), School of Biomedical Engineering (J.L.M., G.P.), Department of Medical Biophysics (A.R.W., R.L.E., A.L.B., G.P.), and Division of Respirology, Department of Medicine (D.G.M., G.P.), Western University, 1151 Richmond St N, London, ON, Canada N6A 5B7; Department of Radiation Oncology, Stanford University School of Medicine, Stanford, Calif (D.P.I.C.); and Department of Physics, Ryerson University, Toronto, Ontario, Canada (M.K.). Received August 6, 2019; revision requested October 30; revision received December 16; accepted December 23. Address correspondence to G.P. (e-mail: gparraga@robarts.ca).

Supported by an Ontario Graduate Scholarship (J.L.M.), a Natural Sciences and Engineering Research Council of Canada postdoctoral fellowship (D.P.I.C.) and doctoral award (R.L.E.), an Ontario Graduate Scholarship and a Canadian Institutes of Health Research Canada Graduate Scholarship (A.L.B.), the Parker B. Francis Fellowship Program and Canada Research Chair Program (tier 2) (M.K.), and the Natural Sciences and Engineering Research Council of Canada, Canada Research Chair Program (tier 1), and Canadian Institutes of Health Research (G.P.).
\end{abstract}

Conflicts of interest are listed at the end of this article.

Radiology 2020; 00:1-10 • https://doi.org/10.1148/radiol.2020191735 • Content code: CH

Background: Pulmonary imaging of chronic obstructive pulmonary disease (COPD) has focused on CT or MRI measurements, but these have not been evaluated in combination.

Purpose: To generate multiparametric response map (mPRM) measurements in ex-smokers with or without COPD by using volume-matched CT and hyperpolarized helium $3\left({ }^{3} \mathrm{He}\right)$ MRI.

Materials and Methods: In this prospective study (https://clinicaltrials.gov, NCT02279329), participants underwent MRI and CT and completed pulmonary function tests, questionnaires, and the 6-minute walk test between December 2010 and January 2019. Disease status was determined by using Global initiative for chronic Obstructive Lung Disease (GOLD) criteria. The mPRM voxel values were generated by using co-registered MRI and CT labels. Kruskal-Wallis and Bonferroni tests were used to determine differences across disease severity, and correlations were determined by using Spearman coefficients.

Results: A total of 175 ex-smokers (mean age, 69 years \pm 9 [standard deviation], 108 men) with or without COPD were evaluated. Ex-smokers without COPD had a larger fraction of normal mPRM voxels (60\% vs $37 \%, 20 \%$, and $7 \%$ for GOLD I, II, and III/ IV disease, respectively; all $P \leq .001$ ) and a smaller fraction of abnormal voxels, including small airways disease (normal CT, not ventilated: $5 \%$ vs $6 \%$ [not significant], $11 \%$, and $19 \%$ [P $\leq .001$ for both] for GOLD I, II, and III/IV disease, respectively) and mild emphysema (normal CT, abnormal apparent diffusion coefficient [ADC]: $33 \%$ vs 54\%, 56\%, and 54\% for GOLD I, II, and III/IV disease respectively; all $P \leq .001)$. Normal mPRM measurements were positively correlated with forced expiratory volume in 1 second $\left(\mathrm{FEV}_{1}\right)(r=0.65, P<.001)$, the $\mathrm{FEV}_{1}$-to-forced vital capacity ratio $(r=0.81, P<.001)$, and diffusing capacity $(r=$ $0.75, P<.001)$ and were negatively correlated with worse quality of life $(r=-0.48, P<.001)$. Abnormal mPRM measurements of small airways disease (normal CT, not ventilated) and mild emphysema (normal CT, abnormal ADC) were negatively correlated with $\mathrm{FEV}_{1}(r=-0.65$ and -0.42 , respectively; $P<.001)$ and diffusing capacity $(r=-0.53$ and -0.60 , respectively; $P<.001)$ and were positively correlated with worse quality of life $(r=0.45$ and $r=0.33$, respectively; $P<.001)$, both of which were present in ex-smokers without COPD.

Conclusion: Multiparametric response maps revealed two abnormal structure-function results related to emphysema and small airways disease, both of which were unexpectedly present in ex-smokers with normal spirometry and CT findings.

(C) RSNA, 2020

Online supplemental material is available for this article.

$I^{n}$

n patients with chronic obstructive pulmonary disease (COPD), irreversible airflow obstruction related to parenchymal destruction, airway remodeling, and luminal obstruction (1) drives debilitating symptoms, poor quality of life, and premature death. While forced expiratory volume in 1 second $\left(\mathrm{FEV}_{1}\right)$ remains a key clinical and diagnostic measurement of COPD, it cannot provide regional information, nor can it be used to adequately discriminate between patients with different underlying diseases, such as airspace enlargement and small airways disease, that manifest differently in individual patients.

To address some of the limitations of spirometry measurements, thoracic $\mathrm{x}$-ray CT has yielded quantitative airway (2) and parenchyma (3) information, as well as indirect information related to small airways disease generated using parametric response maps (4-6). Pulmonary CT has been used widely in studies such as the Genetic Epidemiology of Chronic Obstructive Pulmonary Disease, or COPDGene (7), Subpopulations and Intermediate Outcome Measures in COPD, or SPIROMICS (8), Multi-Ethnic Study of Atherosclerosis, or MESA (9), Canadian Cohort Obstructive Lung Disease, or CANCold (10), and Evaluation of COPD Longitudinally to Identify Predictive Surrogate Endpoints, or ECLIPSE (11). CT parametric response maps are used to quantify COPD severity $(4,12)$, monitor

This copy is for personal use only. To order printed copies, contact reprints@rsna.org 


\section{Abbreviations}

$\mathrm{ADC}=$ apparent diffusion coefficient, $\mathrm{COPD}=$ chronic obstructive pulmonary disease, DLco = diffusing capacity of the lung for carbon monoxide, $\mathrm{FEV}_{1}=$ forced expiratory volume in 1 second, $\mathrm{FVC}=$ forced vital capacity, GOLD = Global initiative for chronic Obstructive Lung Disease, $\mathrm{mPRM}=$ multiparametric response map, $\mathrm{SGRQ}=$ St George's Respiratory Questionnaire, TINCan = Thoracic Imaging Network of Canada

\section{Summary}

Multiparametric response maps derived from CT and hyperpolarized helium 3 MRI revealed emphysema and small airways disease not otherwise identified with CT or MRI alone.

\section{Key Results}

- Combined CT and MRI multiparametric response maps (mPRMs) reflected chronic obstructive pulmonary disease (COPD) severity.

- The fraction of lung occupied by normal mPRM voxels was $60 \%$ in ex-smokers without and 7\% in those with Global initiative for chronic Obstructive Lung Disease III/IV (severe to very severe) COPD $(P<.001)$.

- Ex-smokers without COPD had patterns of subclinical emphysema (normal CT and ventilation with abnormal apparent diffusion coefficients) and small airways disease (normal CT but not ventilated).

COPD progression $(13,14)$, and predict survival after transplantation (6).

Hyperpolarized noble gas MRI also has been used to measure regional lung ventilation (15) and airspace enlargement $(16,17)$ in patients with COPD. MRI ventilation heterogeneity is quantified by using ventilation defect percentage, which is related to COPD severity and exacerbation (18). Hyperpolarized helium $3\left({ }^{3} \mathrm{He}\right) \mathrm{MRI}$ apparent diffusion coefficients (ADCs) may also be used in patients with COPD to estimate terminal airspace enlargement (17), and such measurements have been histologically validated (19).

The Thoracic Imaging Network of Canada (TINCan) (20) study performed volume-matched CT and MRI in ex-smokers with or without COPD in whom spatial correlations between MRI ventilation defects and nonenhanced low-dose CT measurements of emphysema and small airways disease were reported (18). CT parametric response map analysis in TINCan participants also revealed spatial correlations between parametric response map measurements of emphysema and gas trapping with MRI values (21). In addition, in the TINCan study, in a small group of at-risk ex-smokers without CT evidence of emphysema and with modestly abnormal diffusing capacity, there was abnormal MRI ADC, profound exercise limitation, and diminished quality of life (22). Given the complementary nature of pulmonary CT and hyperpolarized gas MRI, we postulated that combining the information obtained with these two modalities would yield new measures of lung abnormality not seen when using CT or MRI alone. On the basis of these findings, we hypothesized that by combining MRI and CT measurements using a multiparametric approach, phenotypes would be observed that cannot be detected using CT or MRI alone. The purpose of this study was to generate multiparametric response map (mPRM) measurements in ex-smokers with or without
COPD using sequentially acquired volume-matched CT and hyperpolarized ${ }^{3} \mathrm{He}$ MRI measurements.

\section{Materials and Methods}

\section{Study Participants and Design}

We evaluated ex-smokers with or without COPD who provided written informed consent to participate in a research ethics board-approved and Health Insurance Portability and Accountability Act-compliant registered study (https:// clinicaltrials.gov, NCT02279329). Participants were recruited from local tertiary care clinics and through advertisements as a convenience sample. Patients were included in the study if they were men or women aged 45-90 years with a clinical diagnosis of COPD or a smoking history of more than 10 pack-years who were in stable health and were ambulatory. Exclusion criteria included contraindications to MRI, missing complete CT and MRI data sets, or diagnosis of bronchiectasis only (Fig 1). The study was prospectively planned and performed from December 2010 to January 2019; participants underwent one 2-hour visit and completed spirometry, plethysmography, quality-oflife questionnaires, exercise capacity tests, and MRI and CT examinations, as previously described (20). Evaluations were performed 20 minutes after administration of four $100-\mu \mathrm{g}$ doses of novo-salbutamol hydrofluoroalkane via a metereddose inhaler (Teva Novopharm, Toronto, Ontario, Canada) through a spacer (AeroChamber Plus; Trudell Medical International, London, Ontario, Canada). Participants in the TINCan study have been previously described $(18,22-26)$. These prior articles dealt with cross-sectional and longitudinal analyses of CT and MRI data and their relationships with quality of life, exacerbations, and exercise capacity, whereas in this article we report on the development of an mPRM approach and apply this to 175 TINCan participants who had the complete image data sets required for analysis. Data generated during this study are available from the corresponding author, and the mPRM method we developed is available online (www. imaging.robarts. calparragal).

\section{Pulmonary Function and Quality-of-Life Tests}

Spirometry and plethysmography were performed and diffusing capacity of the lung for carbon monoxide (DLco ) was measured and evaluated according to the American Thoracic Society and European Respiratory Society guidelines (27-29) using a body plethysmograph (MedGraphics Elite Series; MGC Diagnostic, St Paul, Minn) with an attached gas analyzer. The St. George's Respiratory Questionnaire (SGRQ) was administered (30), and a 6-minute walk test was performed (31).

\section{MRI Examination}

All MRI examinations were performed using previously described methods (20). Briefly, anatomic proton $\left({ }^{1} \mathrm{H}\right)$, hyperpolarized ${ }^{3} \mathrm{He}$ static ventilation, and diffusion-weighted MRI were performed with the patient in the supine position in a 3.0-T Discovery MR750 system (GE Healthcare, Milwaukee, Wis) with broadband imaging capabilities. The ${ }^{1} \mathrm{H}$ MRI examination was performed using a fast spoiled gradient-recalled- 


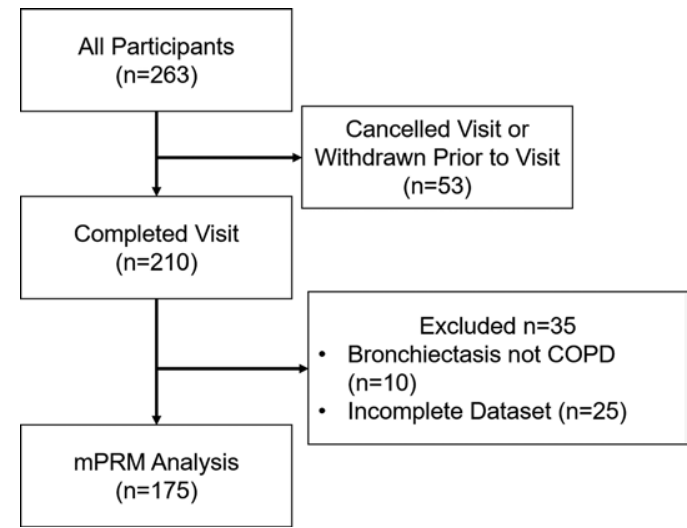

Figure 1: Flowchart shows the participants enrolled in the study. $\mathrm{COPD}=$ chronic obstructive pulmonary disease, mPRM = multiparametric response map.

echo sequence (repetition time msec/echo time msec, 4.7/1.2; flip angle, $30^{\circ}$ ) with a whole-body radiofrequency coil during breath holding after inhalation of $1.0 \mathrm{~L}$ of nitrogen $\left(\mathrm{N}_{2}\right)$.

The ${ }^{3} \mathrm{He}$ polarization was performed to $30 \%-40 \%$ polarization, and all ${ }^{3} \mathrm{He}$ MRI examinations were performed using a single-channel rigid elliptical transmit-receive chest coil (RAPID Biomedical, Wuerzburg, Germany) during a breath hold of 1.0 $\mathrm{L}$ of diluted hyperpolarized gas. The ${ }^{3} \mathrm{He}$ static ventilation was performed using a fast spoiled gradient-recalled-echo sequence (4.3/1.4; flip angle, $7^{\circ}$ ) while diffusion-weighted ${ }^{3} \mathrm{He}$ was performed with a fast spoiled gradient-recalled-echo sequence (7.6/3.7; flip angle, $8^{\circ}$ ) with and without diffusion sensitization with a $b$ value of $1.6 \mathrm{sec} / \mathrm{cm}^{2}$. A detailed description of the MRI acquisition parameters is provided in Appendix E1 (online).

\section{CT Examination}

Within 30 minutes of MRI, CT was performed with the patient in the supine position in a 64-slice Lightspeed VCT scanner (GE Healthcare) during a breath hold after inhalation of $1.0 \mathrm{~L}$ of $\mathrm{N}_{2}$ from functional residual capacity using the following parameters: $64 \times 0.625 \mathrm{~mm}$ collimation, $120 \mathrm{kVp}$, 100-mA effective current, 500-msec rotation time, and 1.0 pitch (22). Specific CT acquisition parameters are provided in Appendix E1 (online). Total effective dose was estimated as $1.8 \mathrm{mSv}$ using the ImPACT CT patient dosimetry calculator (based on Health Protection Agency [UK] NRBP-SR250).

\section{Image Analysis}

MRI and CT image segmentations were performed by an observer (J.L.M., 1.5 years of experience). The ${ }^{3} \mathrm{He}$ and proton $\left({ }^{1} \mathrm{H}\right)$ MRI scans were semiautomatically segmented and coregistered using in-house software (21). Diffusion-weighted images were automatically processed to generate lung ADC images (21). CT images were segmented using Pulmonary Workstation 2.0 software (VIDA Diagnostics, Coralville, Iowa). Image registration of ${ }^{3} \mathrm{He}$ ventilation, $\mathrm{ADC}$, and $\mathrm{CT}$ images was performed using a custom algorithm in MATLAB R2018a software (Mathworks, Natick, Mass). CT and ${ }^{1} \mathrm{H}$ MRI were nonrigidly registered using the modality-independent neighborhood descriptor method (32); ${ }^{3} \mathrm{He}$ ventilation and
ADC images were co-registered using intensity-based rigid registration. Image analysis specifics are provided in Appendix E1 (online).

\section{Development of mPRMs}

Once all the images were co-registered, voxels within the proton thoracic cavity were labeled to generate mPRMs. These labels were generated based on ventilation, ADC values, and CT density histogram threshold values, as shown in Figure 2. The CT threshold of $-950 \mathrm{HU}$ was used as a well-established and validated measure of emphysema in the lungs (3), whereas the ADC threshold of $0.30 \mathrm{~cm}^{2} / \mathrm{sec}$ was based on experimentally acquired ADC values reported in a group of elderly participants who had never smoked. The threshold we used was equal to 1 standard deviation of the mean of this group, as a conservative estimate. ADC values for this group of participants who had never smoked were previously reported (33). Although CT was performed at functional residual capacity plus $1 \mathrm{~L}$, this lung volume is roughly $80 \%-90 \%$ of total lung capacity in patients with COPD, especially when lying supine in the scanner; moreover, Madani and colleagues (34) also previously reported that such differences related to lung volume are within a few percentage points for relative area of the lung of less than -950 HU values and that these are unlikely to be clinically relevant. mPRMs were evaluated by an observer (J.L.M.). Figure 3 shows the generation of mPRM values and the five resultant MPRM voxel classifications: (a) normal (green); (b) ventilated, CT greater than or equal to -950 HU, abnormal ADC (yellow); (c) ventilated, CT emphysema, abnormal ADC (orange); (d) not ventilated, CT greater than or equal to $-950 \mathrm{HU}$, no $\mathrm{ADC}$ (red); and (e) not ventilated, CT emphysema, no ADC (burgundy). ADC values reflect gas displacement but only in ventilated regions of the lung. Because CT and MRI scans were acquired at the same lung volume, the potential for co-registration errors is low, but errors are not impossible. Hence, voxels labeled ventilated, CT emphysema, normal ADC were classified as registration errors because of the high sensitivity of MRI to emphysema, which suggests that normal ADC and CT emphysema are unlikely in the same voxel, although this finding may also stem from partial volume averaging.

\section{Statistical Analyses}

Statistical tests were performed using statistical software (SPSS Statistics 25.0; SPSS, Armonk, NY). A Shapiro-Wilk test was used to determine normality of the data. Kruskal-Wallis tests with post hoc Dunn-Bonferroni correction were used to determine differences between groups for mPRM voxel distributions. Spearman correlation coefficients with a Bonferroni correction were determined for mPRM voxel distributions, pulmonary function, and quality-of-life test measurements. Since each clinical measurement was correlated multiple times with every mPRM classification measurement, a Bonferroni correction of six was multiplied to the original $P$ values, and the adjusted $P$ values were compared for significance. All results were considered significant when the probability of making a type I error was less than $5 \%(P<.05)$. 
Results

\section{Study Participants}

Participants were aged 48-87 years (mean age, 69 years \pm 9 [standard deviation]; mean age of men, 70 years \pm 10 ; age range of men, 48-87 years; mean age of women, 68 years \pm 8 ; age range of women, 52-83 years). We generated mPRM measurements (Figs 2, $3)$ in 175 ex-smokers without $(n=67$; mean age, 68 years \pm 10; 39 men) or with Global initiative for chronic Obstructive Lung Disease (GOLD) grade $\mathrm{I}(n=24 ;$ mean age, 74 years $\pm 7 ; 20$ men), grade II $(n=49$; mean age, 69 years \pm $8 ; 29$ men), or grade III/IV ( $n$ $=35$; mean age, 68 years \pm 9 ; 20 men) COPD. Mean ADC in the participants who never smoked was $0.26 \mathrm{~cm}^{2} / \mathrm{sec} \pm$ 0.04, which was consistent with previous work (35) and was used to determine the abnormal ADC threshold. Figure 1 shows that of the 263 total participants enrolled, 53 withdrew prior to the first visit; 10 had bronchiectasis, not COPD; and 25 did not complete imaging (either MRI or CT); thus, 175 participants were evaluated.

Table 1 provides participant characteristics, pulmonary function test results, and imaging and quality-of-life measurements for all participants and for participants classified by subgroup according to GOLD criteria, which were different across subgroups (all $P \leq .05$ ). Figure 4 shows representative ${ }^{3} \mathrm{He}$ ventilation MRI scans, as well as ADC and CT maps showing relative area of the lung with attenuation less than $-950 \mathrm{HU}$. Figure 4 also shows there was qualitatively worse or increased MRI ventilation heterogeneity, ADC, and voxels with attenuation less than $-950 \mathrm{HU}$ with increasing COPD severity.

\section{mPRM Lung Fraction Measurements}

Figure 5 shows the resultant mPRM findings for all participants by lung fraction and representative center-slice coronal mPRM maps for representative participants who were ex-smokers with or without COPD. Figure $5, A$, shows there was a larger fraction of normal mPRM voxels in ex-smokers (all $P \leq .001$ ) and a smaller fraction of abnormal mPRM voxels compared with all subgroups of participants with COPD. Ex-smokers without COPD also reported a smaller fraction of unventilated normal CT voxels compared with ex-smokers with GOLD II or III/IV COPD (all $P<.001$ ). The mPRM analysis revealed two novel mPRM voxel types, both of which were present in otherwise healthy ex-smokers, and which reflect mild emphysema or hy- perinflation and small airways disease, respectively. Figure 5, $B$, also shows there was a greater fraction of abnormal mPRM voxels as COPD severity increased.

\section{mPRM Relationships with Pulmonary Measurements}

Table 2 shows Spearman correlation coefficients for mPRM lung fraction measurements with $\mathrm{FEV}_{1}$ percentage predicted, FEV ${ }_{1}$-to-forced vital capacity (FVC) ratio (hereafter, $\mathrm{FEV}_{1}$ /FVC), DLco, SGRQ total score, and 6-minute walk distance in ex-smokers with or without COPD. In all participants, normal mPRM lung fraction measurements (green voxels in Figs 3, 5) were correlated with $\mathrm{FEV}_{1}$ percentage predicted $(r=0.65, P<.001), \mathrm{FEV}_{1} / \mathrm{FVC}(r=0.81, P<$ $.001)$, DLco $(r=0.75, P<.001)$, and SGRQ $(r=-0.48$, $P<.001)$. The mPRM ventilated, normal CT, and abnormal ADC (yellow voxels) lung fraction measurements were correlated with $\mathrm{FEV}_{1}$ percentage predicted $(r=-0.42, P<.001)$, $\mathrm{FEV}_{1} / \mathrm{FVC}(r=-0.60, P<.001)$, DLco $(r=-0.60, P<$ $.001)$, and SGRQ $(r=0.33, P<.001)$. Table 2 also shows that mPRM ventilated, CT emphysema, and abnormal ADC (orange voxels) lung fraction measurements were correlated with $\mathrm{FEV}_{1}$ percentage predicted $(r=-0.64, P<.001), \mathrm{FEV}_{1} / \mathrm{FVC}$ $(r=-0.83, P<.001)$, DLco $(r=-0.66, P<.001)$, and SGRQ $(r=0.45, P<.001)$. Unventilated with normal CT (red voxels in Figs 3, 5) mPRM lung fraction measurements were correlated with $\mathrm{FEV}_{1}$ percentage predicted $(r=-0.65$, $P<.001), \mathrm{FEV}_{1} / \mathrm{FVC}(r=-0.70, P<.001)$, DLco $(r=-0.53$, $P<.001)$, and SGRQ $(r=0.45, P<.001)$ (Figs 3, 5), whereas unventilated CT emphysema (burgundy voxels) lung fraction 


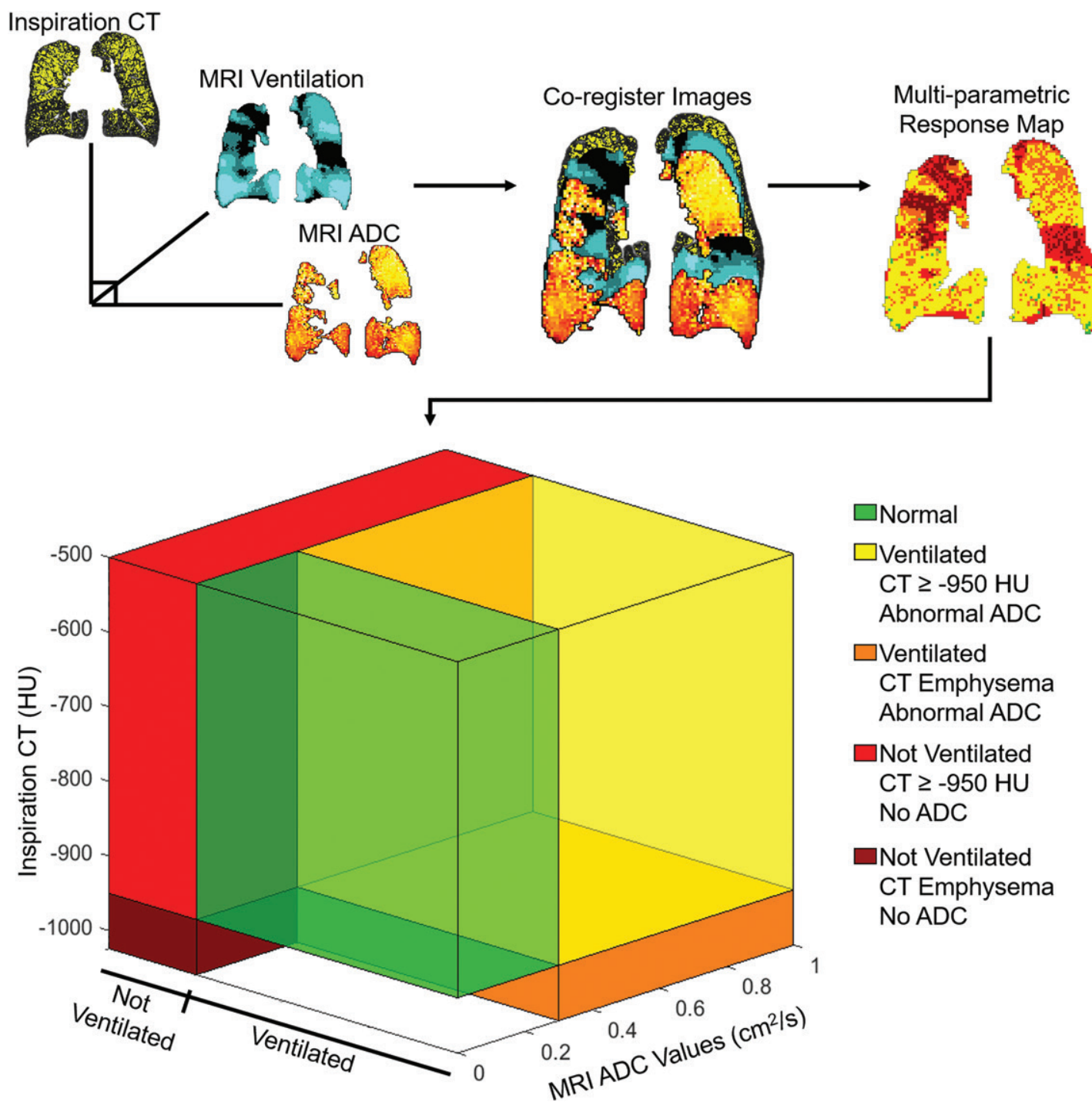

Figure 3: Schematic for multiparametric (MRI and CT) response maps in the setting of chronic obstructive pulmonary disease (COPD). Top: Co-registration of three contemporaneously acquired images in an ex-smoker with Global initiative for chronic Obstructive Lung Disease III/IV COPD (CT, MRI ventilation, MRI apparent diffusion coefficient [ADC]) into one map. Bottom: Threedimensional multiparametric response map voxel classification. Green = ventilated voxels, $C T \geq-950 \mathrm{HU}$, normal $A D C$; yellow $=$ ventilated voxels, $C T \geq-950 \mathrm{HU}$, and abnormal $\mathrm{ADC}\left(>0.30 \mathrm{~cm}^{2} / \mathrm{sec}\right)$; orange = ventilated voxels, CT emphysema, and abnormal $A D C$; red = not ventilated, $C T \geq-950 \mathrm{HU}$, no measurable $A D C$; burgundy = not ventilated, $C T$ emphysema, no measurable $A D C$; clear = registration error.

measurements were correlated with $\mathrm{FEV}_{1}$ percentage predicted $(r=-0.72, P<.001), \mathrm{FEV}_{1} / \mathrm{FVC}(r=-0.86, P<.001)$, DLco $(r=-0.63, P<.001)$, and SGRQ $(r=0.46, P<.001)$. Table 2 also shows that all mPRM lung fraction measurements except for ventilated, normal CT, and abnormal ADC were correlated with 6-minute walk distance. Figures E1-E5 (online) show univariate regressions for mPRM lung fraction and clinical measurements, while Table E1 (online) provides Spearman correlation coefficients for all disease severity subgroups.

\section{mPRM Phenotypes in Ex-Smokers without COPD}

Figure 6 shows mPRMs for four representative ex-smokers with normal spirometry and CT results. These maps highlight the heterogeneity observed in ex-smokers and the regional distribution of mPRM voxels, including (a) normal ventilation, $\mathrm{CT}$, and $\mathrm{ADC}$; (b) normal ventilation and $\mathrm{CT}$ but abnormal ADC; and (c) nonventilated and normal CT. For example, Figure 6, $A$, shows a resultant or normal mPRM map in a 51 -year-old man with a 32 pack-year smoking history, normal spirometry results $\left(\mathrm{FEV}_{1}=75 \%\right.$ predicted, $\left.\mathrm{FEV}_{1} / \mathrm{FVC}=0.77\right)$, and normal DLco (95\% predicted). In contrast, in Figure $6, B$, there is a mixture of (a) normal ventilation, CT, and ADC and (b) normal ventilation and CT but abnormal ADC in a 76-year-old man with a 24 pack-year smoking history, normal spirometry results $\left(\mathrm{FEV}_{1}=\right.$ $115 \%$ predicted, $\mathrm{FEV}_{1} / \mathrm{FVC}=0.72$ ), and abnormal DLco (59\% predicted). Figure 6, $C$, is an mPRM map obtained in an 80-year-old woman with a 27 pack-year smoking history, similar pulmonary function measurements $\left(\mathrm{FEV}_{1}=\right.$ $124 \%$ predicted, $\mathrm{FEV}_{1} / \mathrm{FVC}=0.71$ ), and abnormal DLco 
Table 1: Participant Characteristics

\begin{tabular}{|c|c|c|c|c|c|}
\hline Parameter & $\begin{array}{l}\text { All } \\
(n=175)\end{array}$ & $\begin{array}{l}\text { Ex-smokers without COPD } \\
(n=67)\end{array}$ & $\begin{array}{l}\text { GOLD I } \\
(n=24)\end{array}$ & $\begin{array}{l}\text { GOLD II } \\
(n=49)\end{array}$ & $\begin{array}{l}\text { GOLD III/IV } \\
(n=35)\end{array}$ \\
\hline Age (y) & $69 \pm 9$ & $68 \pm 10$ & $74 \pm 7$ & $69 \pm 8$ & $68 \pm 9$ \\
\hline Men* & $108(62)$ & $39(58)$ & $20(83)$ & $29(59)$ & $20(57)$ \\
\hline BMI $\left(\mathrm{kg} / \mathrm{m}^{2}\right)$ & $28 \pm 5$ & $29 \pm 4$ & $28 \pm 4$ & $26 \pm 4$ & $26 \pm 5$ \\
\hline No. of pack-years & $40 \pm 28$ & $28 \pm 18$ & $48 \pm 34$ & $46 \pm 28$ & $53 \pm 30$ \\
\hline $\mathrm{FEV}_{1}\left(\%_{\text {pred }}\right)$ & $77 \pm 28$ & $98 \pm 19$ & $95 \pm 11$ & $64 \pm 9$ & $35 \pm 7$ \\
\hline FVC $\left(\%_{\text {pred }}\right)$ & $91 \pm 19$ & $92 \pm 17$ & $109 \pm 12$ & $92 \pm 15$ & $71 \pm 15$ \\
\hline $\mathrm{FEV}_{1} / \mathrm{FVC}$ & $0.62 \pm 0.18$ & $0.80 \pm 0.06$ & $0.63 \pm 0.05$ & $0.53 \pm 0.09$ & $0.38 \pm 0.08$ \\
\hline TLC $\left(\%_{\text {pred }}\right)$ & $111 \pm 18$ & $101 \pm 14$ & $112 \pm 12$ & $115 \pm 18$ & $125 \pm 17$ \\
\hline RV/TLC $\left(\%_{\text {pred }}\right)$ & $121 \pm 27$ & $107 \pm 18$ & $107 \pm 17$ & $125 \pm 18$ & $161 \pm 23$ \\
\hline $\mathrm{R}_{\mathrm{AW}}\left(\%_{\text {pred }}\right)$ & $170 \pm 140(n=173)$ & $115 \pm 66$ & $122 \pm 44$ & $175 \pm 112$ & $314 \pm 218(n=33)$ \\
\hline DLco $\left(\%_{\text {pred }}\right)$ & $65 \pm 23(n=173)$ & $79 \pm 21$ & $69 \pm 19$ & $57 \pm 18(n=48)$ & $42 \pm 18(n=34)$ \\
\hline $\mathrm{RA}_{950}(\%)$ & $6 \pm 9$ & $1 \pm 1$ & $4 \pm 4$ & $8 \pm 9$ & $16 \pm 11$ \\
\hline VDP (\%) & $12 \pm 10$ & $6 \pm 4$ & $8 \pm 5$ & $14 \pm 8$ & $25 \pm 9$ \\
\hline $\operatorname{ADC}\left(\mathrm{cm}^{2} / \mathrm{sec}\right)$ & $0.35 \pm 0.11$ & $0.26 \pm 0.03$ & $0.34 \pm 0.08$ & $0.39 \pm 0.10$ & $0.46 \pm 0.09$ \\
\hline SGRQ total & $35 \pm 22(n=165)$ & $25 \pm 22(n=61)$ & $23 \pm 16(n=22)$ & $38 \pm 15(n=48)$ & $58 \pm 13(n=34)$ \\
\hline 6MWD (min) & $393 \pm 87(n=163)$ & $405 \pm 91(n=65)$ & $420 \pm 50$ & $399 \pm 88(n=46)$ & $340 \pm 83(n=28)$ \\
\hline
\end{tabular}

Note.-Unless otherwise indicated, data are mean \pm standard deviation and data in parentheses are numbers of patients. ADC $=$ apparent diffusion coefficient, $\mathrm{BMI}=$ body mass index, $\mathrm{COPD}=$ chronic obstructive pulmonary disease, DLco = diffusing capacity of the lung for carbon monoxide, $\mathrm{FEV}_{1}$ = forced expiratory volume in 1 second, $\mathrm{FVC}$ = forced vital capacity, GOLD = Global initiative for chronic Obstructive Lung Disease grade, $\%_{\text {pred }}=$ percentage predicted, $\mathrm{R}_{\mathrm{AW}}=$ airways resistance, $\mathrm{RA}_{950}=$ relative area of the lung $<-950 \mathrm{HU}, \mathrm{RV}$ = residual volume, SGRQ = St George's Respiratory Questionnaire, $6 \mathrm{MWD}=6$-minute walk distance, TLC = total lung capacity, VDP = ventilation defect percentage.

* Data are numbers of participants, and data in parentheses are percentages.

(49\% predicted) that consists of mainly normal ventilation and CT but abnormal ADC. Finally, Figure 6, D, shows an mPRM map in a 72-year-old man with a 20 pack-year smoking history and normal spirometry $\left(\mathrm{FEV}_{1}=122 \%\right.$ predicted, $\left.\mathrm{FEV}_{1} / \mathrm{FVC}=0.80\right)$ and DLco $(73 \%$ predicted $)$ results that consists of a mixture of normal ventilation, CT, and $\mathrm{ADC}$ throughout the lungs and nonventilated, normal $\mathrm{CT}$, and no ADC in wedge-shaped peripheral (subsegmental) regions.

\section{Discussion}

Previous work has shown that hyperpolarized gas MRI and CT provide complementary disease information in the setting of chronic obstructive pulmonary disease (COPD), but this information has not been combined into one image. In this study, we generated multiparametric response map (mPRM) measurements by combining CT and MRI data in 175 exsmokers, including 108 participants with and 67 without COPD. We observed (a) significantly different mPRM lung fraction measurements with increasing disease severity, (b) significant associations for mPRM lung fraction measurements with pulmonary function and quality of life (all $P<.001$ ), and (c) two mPRM voxel types that were suggestive of disease transition or progression in ex-smokers with normal spirometry and CT results, neither of which were detected with CT or MRI alone.

Lung fraction measurements obtained with mPRM were significantly different with increasing COPD severity, which is not surprising given the results of previous single-modality
CT and MRI studies in participants with COPD $(4,21,24,36)$. These previous studies also showed that as COPD severity increased, there was a decrease in total airway count and terminal bronchioles $(24,36)$. With increasing COPD severity, mPRM measurements also transition from (a) mainly normal voxels to (b) hyperinflated or mild emphysema voxels to (c) ventilation abnormalities and then to $(d)$ advanced emphysema with ventilation abnormalities.

These mPRM lung fraction measurements were also correlated with pulmonary function, quality of life, and exercise capacity. The relationship of mPRM phenotypes with DLco appears to transition from potential subclinical emphysema in ex-smokers with normal spirometry and CT results to progressively more severe emphysema in the setting of COPD. These results are consistent with previous findings of the strong relationship between DLco and measures of emphysema $(2,3,22)$.

By identifying abnormal lung regions using mPRM maps, there is the potential to provide insight into the transition from normal to diseased COPD states, but this hypothesis requires testing in a prospective longitudinal study. The heterogeneity of mPRM maps in at-risk ex-smokers shows the presence of abnormal lung regions that are not obvious on CT or MRI scans alone. Voxels with normal CT and ventilation but an abnormal $\mathrm{ADC}$ can be interpreted in the context of previous work that revealed MRI evidence of subclinical airspace enlargement (due to emphysema or alveolar distension) in a small group of ex-smokers without CT maps showing relative area of the lung with attenuation less than $-950 \mathrm{HU}$, consistent with emphysema (22). Regions of elevated ADC may indicate regions of subclinical 
emphysema, possible alveolar distention, or gas trapping and are consistent with previous findings $(21,22)$. While these microstructural changes are not visible on CT images or reflected with spirometry, they may be an indicator of early terminal airspace destruction.

Another key finding in otherwise healthy exsmokers is regions of the lung that have normal CT voxels but no gas distribution. These regions are consistent with subsegmental small airways disease. In the setting of COPD, the narrowing or destruction of small airways is thought to precede the development of emphysema (36), but the affected small airways cannot be detected with spirometry and are invisible on CT $(14,37)$. CT parametric response maps have also provided a way to regionally identify gas trapping based on voxels that appear normal on inspiration CT but abnormal $(<856 \mathrm{HU})$ on expiration CT $(4,14)$, and it has been validated as an imaging measurement of small airways disease in the setting of very severe COPD (38). Multimodality mPRMs unite structural and functional abnormalities in a way that resembles CT parametric response map measurements of emphysema and small airways disease and may provide another way to highlight the transition phases in the setting of COPD. Further evaluation of unventilated normal CT voxels is required with CT airway and parametric response map measurements to clarify the pathophysiology responsible for these findings.

We expected to observe overlap of structure and function imaging findings in participants with COPD. In other words, the mPRM findings in patients with GOLD grades IIV COPD are in keeping with our understanding of COPD
${ }^{3} \mathrm{He} \mathrm{MRI} A D C$
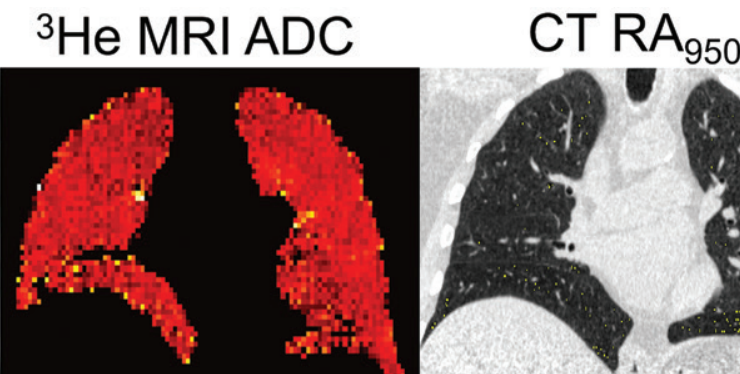

950

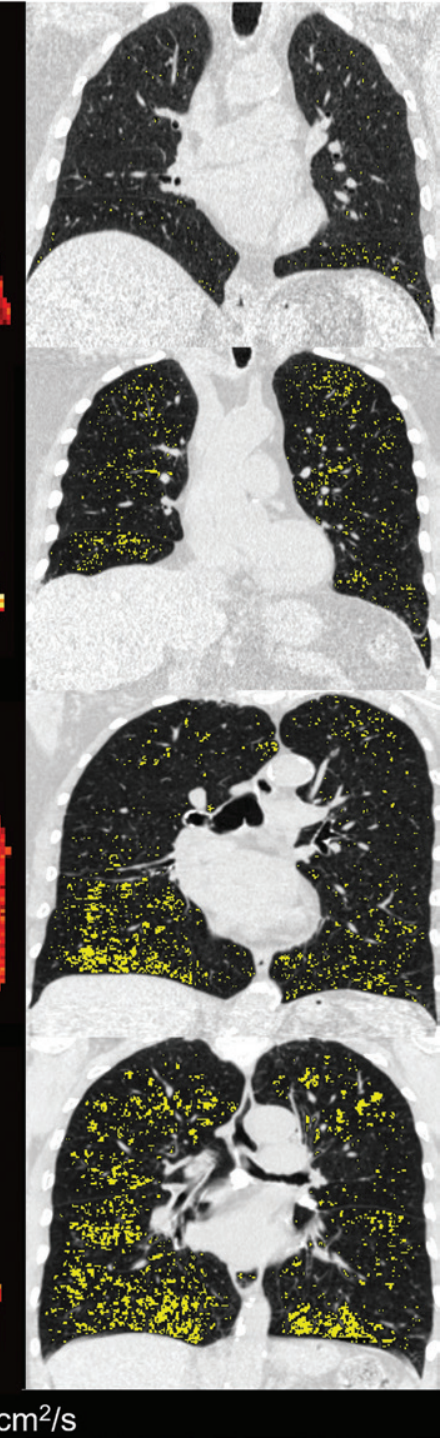

Figure 4: Representative coronal MRI and CT scans by chronic obstructive pulmonary disease (COPD) severity. Ventilation is shown in blue. Yellow shows relative area of the lung with CT attenuation less than $-950 \mathrm{HU}\left(\mathrm{RA}_{950}\right.$ ) on inspiratory CT. The ex-smoker without COPD was a 56-year-old man with a 41 pack-year smoking history and normal pulmonary function (forced expiratory volume in 1 second $\left[\mathrm{FEV}_{1}\right]=101 \%, \mathrm{FEV} /$ forced vital capacity [FVC] ratio $=0.89$, residual volume [RV]/total lung capacity $[\mathrm{TLC}]$ ratio $=106 \%$, diffusing capacity of the lung for carbon monoxide [DLCO] $=79 \%$; all are percentage predicted). The subject with Global initiative for chronic Obstructive Lung Disease (GOLD I) disease was an 87-year-old man with a 15 pack-year smoking history and modestly abnormal pulmonary function $\left(\mathrm{FEV}_{1}=88 \%, \mathrm{FEV}, / \mathrm{FVC}=0.60, \mathrm{RV} / \mathrm{TLC}=95 \%\right.$, DLCO $=59 \%$; all are and abnormal airflow, hyperinflation, and diffusing capacity $(\mathrm{FEV}=66 \%, \mathrm{FEV} / \mathrm{FVC}=0.51, \mathrm{RV} / \mathrm{TLC}=145 \%$, DLCO $=49 \%$; all are percentage predicted). The subject with GOLD III/IV disease was a 76-year-old woman with a 30 pack-year smoking history and highly abnormal airflow, hyperinflation, and highly abnormal diffusing capacity (FEV $=37 \%, F E V_{1} / \mathrm{FVC}=0.52, \mathrm{RV} / \mathrm{TLC}=139 \%$, $\mathrm{DLCO}=19 \%$; all are percentage predicted). ADC = apparent diffusion coefficient. pathophysiology and the finding of increasingly abnormal tissue and small airway structure and function in participants with more advanced or severe COPD. However, on the basis of previous findings, we did not expect to observe mPRM evidence of small airways disease (not ventilated, normal CT) and mild emphysema (ventilated, normal $\mathrm{CT}$, abnormal ADC) in ex-smokers with otherwise normal CT and spirometry values. These two novel findings in a highly heterogeneous convenience sample of ex-smokers 
A

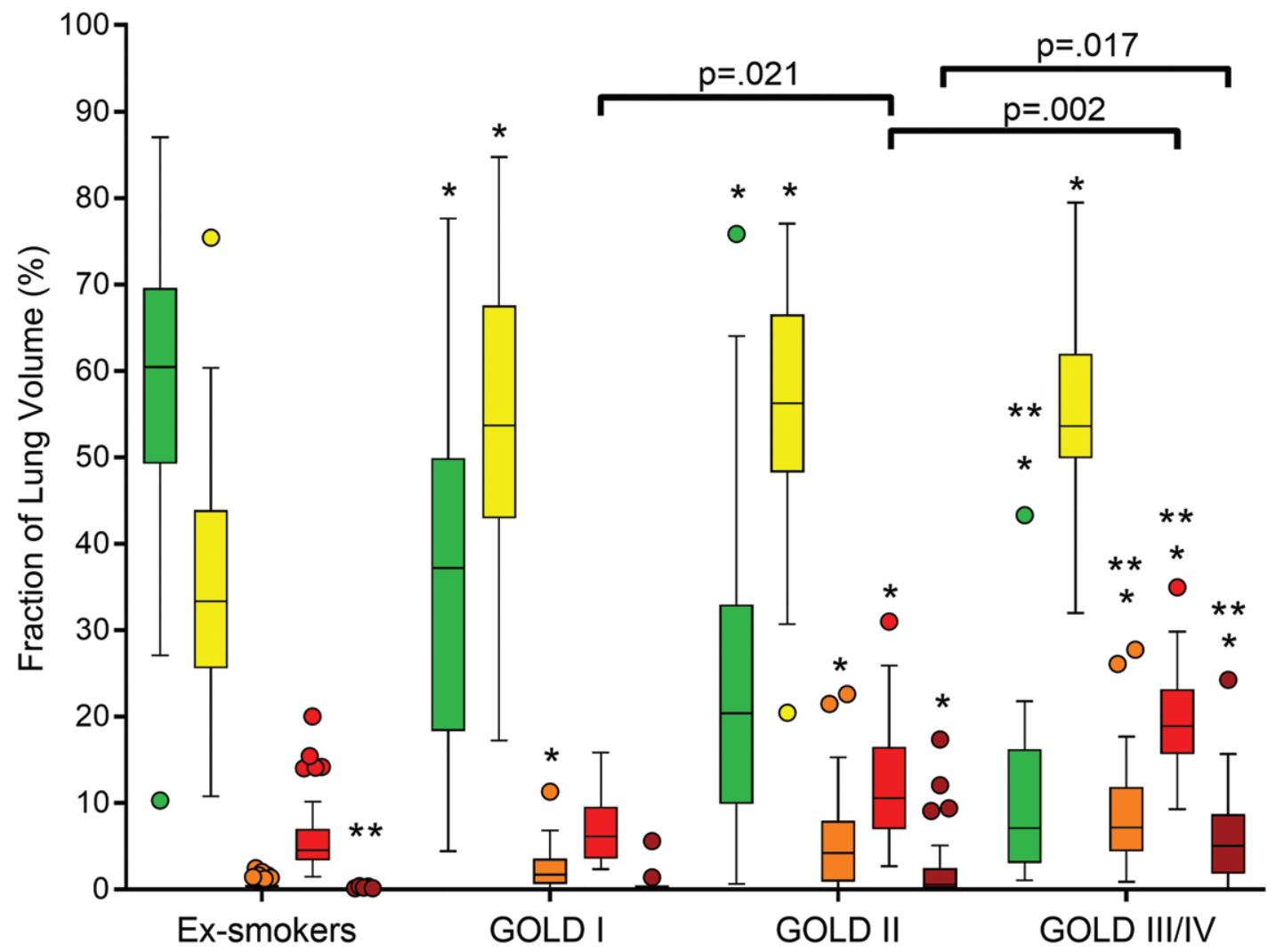

B

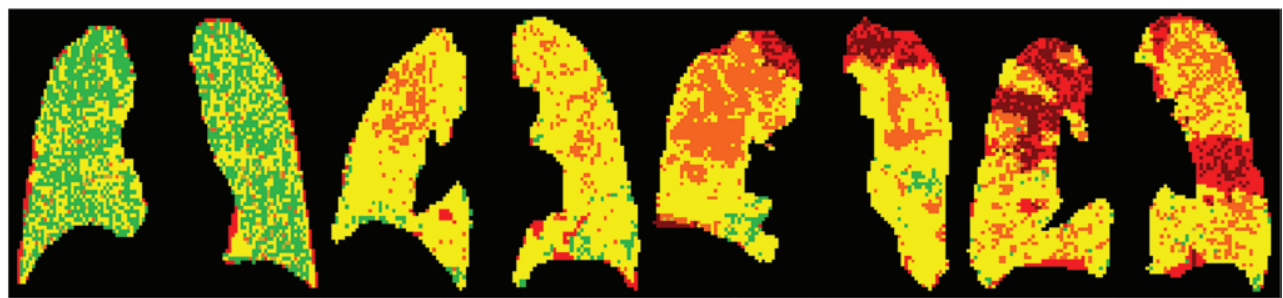

$\square$ Normal $\square$ Ventilated

$\mathrm{CT} \geq-950 \mathrm{HU}$

Abnormal ADC

$\square$ Ventilated

CT Emphysema

Abnormal ADC
Not Ventilated

CT $\geq-950 \mathrm{HU}$ No ADC
Not Ventilated

CT Emphysema No ADC

Figure 5: Multiparametric response map (mPRM) lung fraction measurements and increasing chronic obstructive pulmonary disease (COPD) severity. A, The mPRM voxel category by lung fraction for all ex-smokers without COPD $(n=67)$ and those with Global initiative for chronic Obstructive Lung Disease (GOLD) grade I ( $n=24)$, II ( $n=49)$, or III/IV ( $n=35)$ disease. In ex-smokers with no spirometric evidence of COPD, there is a larger fraction of normal voxels and a smaller fraction of abnormal voxels than in COPD subgroups. As COPD grade increases, there is a decrease in the number of normal voxels and an increase in the number of abnormal voxels. B, Representative mPRM voxel maps in a 79-year-old ex-smoker (32 pack-year smoking history) without COPD (mainly green [normal] and yellow [ventilated, CT $\geq-950 \mathrm{HU}$, abnormal apparent diffusion coefficient $\{A D C\}]$ voxels) and participants with GOLD grade I (71-year-old participant, 66 pack-year smoking history) (mainly yellow, orange [ventilated, CT emphysema, abnormal ADC], and red [not ventilated, CT $\geq-950$ HU] voxels), GOLD grade II (86-year-old participant, 50 pack-year smoking history) (similar to GOLD I except with a greater amount of orange and red voxels), and GOLD grade III/IV (68-year-old participant, 93 pack-year smoking history) (similar to GOLD II except with greater amounts of burgundy [not ventilated, CT emphysema] voxels) disease. *P $\leq .001$ compared with ex-smokers without COPD. ** indicates $P$ $<.01$ compared with GOLD I.

without airflow obstruction challenge our understanding of normal pulmonary function test results in ex-smokers who currently are not considered to have and are not currently undergoing treatment for COPD.

We acknowledge several study limitations, including the fact that MRI and CT co-registration errors will influence voxel classification. Importantly, CT and MRI scans were acquired at the same lung volume, assisting with image registration accuracy (Appendix E1 [online]). ADC images have twice the slice thickness compared with ventilation images, which often makes resampling complex. We also used a fixed threshold for CT emphysema (3) and an experimentally derived fixed ADC threshold (33) based on assumptions about ADC values in agematched participants who never smoked. Other factors $(35,39)$ may influence ADC values in participants with relatively healthy lungs; therefore, the ADC threshold we used might not 
Table 2: Spearman Correlation Coefficients for mPRM Voxel Classes as a Fraction of Total Lung

\begin{tabular}{|c|c|c|c|c|c|}
\hline $\begin{array}{l}\text { Spearman Correlation } \\
\text { Coefficient }(\rho)\end{array}$ & $\begin{array}{l}\text { Normal } \\
\text { (\% of lung) }\end{array}$ & $\begin{array}{l}\text { Ventilated }+ \\
\mathrm{CT} \geq-950 \mathrm{HU}+ \\
\text { Abnormal ADC ( } \% \text { of lung) }\end{array}$ & $\begin{array}{l}\text { Ventilated + CT } \\
\text { Emphysema + Abnormal } \\
\text { ADC ( } \% \text { of lung) }\end{array}$ & $\begin{array}{l}\text { Not Ventilated }+ \\
\mathrm{CT} \geq-950 \mathrm{HU}+ \\
\text { No ADC }(\% \text { of lung })\end{array}$ & $\begin{array}{l}\text { Not Ventilated }+ \text { CT } \\
\text { Emphysema }+ \text { No } \\
\text { ADC ( } \% \text { of lung) }\end{array}$ \\
\hline$\overline{\text { FEV }_{1}\left(\%_{\text {pred }}\right)}$ & $0.65(<.001)^{*}$ & $-0.42(<.001)^{*}$ & $-0.64(<.001)^{*}$ & $-0.65(<.001)^{*}$ & $-0.72(<.001)^{*}$ \\
\hline $\mathrm{FEV}_{1} / \mathrm{FVC}$ & $0.81(<.001)^{*}$ & $-0.60(<.001)^{*}$ & $-0.83(<.001)^{*}$ & $-0.70(<.001)^{*}$ & $-0.86(<.001)^{*}$ \\
\hline $\operatorname{DLco}\left(\%_{\text {pred }}\right)(n=173)$ & $0.75(<.001)^{*}$ & $-0.60(<.001)^{*}$ & $-0.66(<.001)^{*}$ & $-0.53(<.001)^{*}$ & $-0.63(<.001)^{*}$ \\
\hline SGRQ $(n=165)$ & $-0.48(<.001)^{*}$ & $0.33(<.001)^{*}$ & $0.45(<.001)^{*}$ & $0.45(<.001)^{*}$ & $0.46(<.001)^{*}$ \\
\hline 6MWD $(n=163)$ & $0.28(.002)^{*}$ & $-0.19(.08)$ & $-0.27(.003)^{*}$ & $-0.29(.001)^{*}$ & $-0.29(.001)^{*}$ \\
\hline
\end{tabular}

Note.-Data in parentheses are $P$ values and were adjusted using Bonferroni correction of six to account for multiple comparisons for multiparametric response map $(\mathrm{mPRM})$ voxel classes. $\mathrm{ADC}=$ apparent diffusion coefficient, DLco = diffusing capacity of the lung for carbon monoxide, $\mathrm{FEV}_{1}=$ forced expiratory volume in 1 second, $\mathrm{FVC}=$ forced vital capacity, $\%_{\text {pred }}$ = percentage predicted, $6 \mathrm{MWD}=6$-minute walk distance, SGRQ = St George's Respiratory Questionnaire total score.

* Correlations are significant.

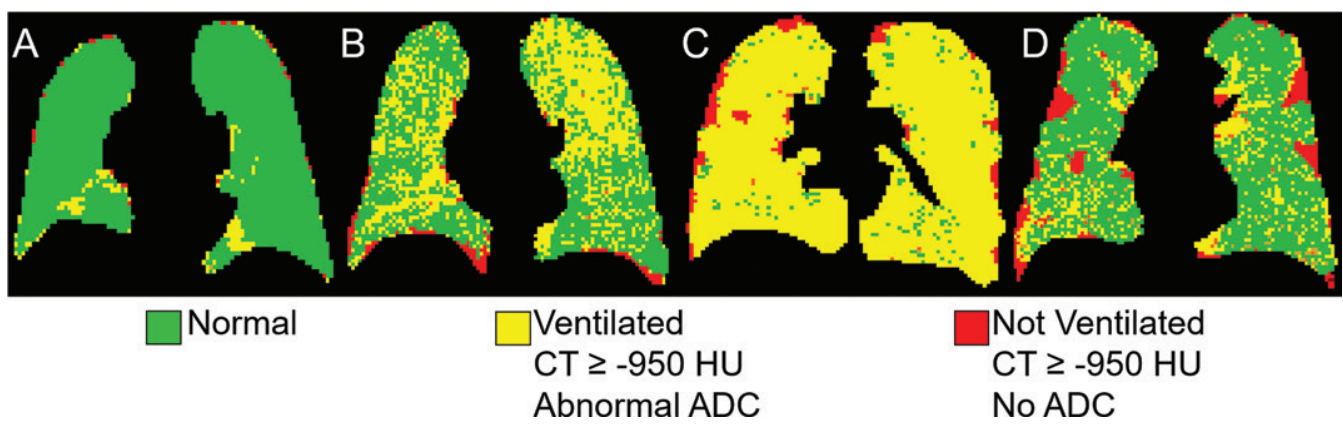

Figure 6: Multiparametric response maps (mPRMs) in representative ex-smokers without chronic obstructive pulmonary disease (COPD). A, Mainly green (normal) voxels in a 51 -year-old man (32 pack-year smoking history, forced expiratory volume in 1 second $\left[\mathrm{FEV}_{1}\right]=75 \%, \mathrm{FEV}_{1} /$ forced vital capacity $[\mathrm{FVC}]=0.77$, diffusing capacity of the lung for carbon monoxide[ $\left.\mathrm{DLCO}\right]=95 \%$ ) B, Mixture of green (normal) and yellow (ventilated, CT $\geq-950 \mathrm{HU}$, abnormal apparent diffusion coefficient [ADC]) voxels in a 76-year-old man (24 pack-year smoking history, FEV $=115 \%, F E V, / F V C=0.72, \mathrm{DLCO}=59 \%$ ). C, Mainly yellow (ventilated, $\mathrm{CT} \geq-950 \mathrm{HU}$, abnormal ADC) voxels in an 80-year-old woman (27 pack-year smoking history, FEV $1=124 \%, F E V_{1} / \mathrm{FVC}=$ $0.71, \mathrm{DLCO}=49 \%$ ). D, Mixture of green (normal), yellow (ventilated, CT $\geq-950 \mathrm{HU}$, abnormal ADC), and red (not ventilated, $\mathrm{CT} \geq-950 \mathrm{HU}$ ) voxels in a 72 -year-old man (20 pack-year smoking history, FEV $=122 \%, \mathrm{FEV}, / \mathrm{FVC}=0.80, \mathrm{DLCO}=73 \%$ ) All percentages are percentage predicted.

represent terminal airspace enlargement or emphysema but instead airspace distention. We acquired CT images at functional residual capacity $+1 \mathrm{~L}$ instead of at full inspiration, for which the $-950-\mathrm{HU}$ threshold is validated (3). For the participants in our study and on the basis of plethysmographic data, functional residual capacity $+1 \mathrm{~L}$ is equal to $80 \%-90 \%$ of total lung capacity, especially when the participant is lying supine in the scanner. This means that the estimates of CT emphysema here should be considered fractionally lower than estimates for patients at full inflation, but this difference is unlikely to be clinically relevant (34). Nevertheless, the emphysema estimates in participants in our study are similar to those in other published studies, such as Multi-Ethnic Study of Atherosclerosis, or MESA (9), Canadian Cohort Obstructive Lung Disease, or CANCold (40), Subpopulations and Intermediate Outcome Measures in COPD, or SPIROMICS (5), and Genetic Epidemiology of Chronic Obstructive Pulmonary Disease, or COPDGene (41). Another limitation of this study was the lack of a control group of age-matched participants who never smoked, which would help provide a better understanding of the physiologic relevance of the mPRM values. Longitudinal data on the lack of progression (or on the progression) of these two new imaging phenotypes of COPD remains to be investigated.

In conclusion, we developed a multimodality image processing pipeline to generate pulmonary multiparametric response map measurements that were related to pulmonary function tests and quality of life and that revealed phenotypes in at-risk ex-smokers that were not detected with CT or MRI alone. To our knowledge, this is the first multiparametric analysis of thoracic CT and MRI in patients with chronic obstructive pulmonary disease and at-risk ex-smokers.

Author contributions: Guarantor of integrity of entire study, G.P.; study concepts/ study design or data acquisition or data analysis/interpretation, all authors; manuscript drafting or manuscript revision for important intellectual content, all authors; approval of final version of submitted manuscript, all authors; agrees to ensure any questions related to the work are appropriately resolved, all authors; literature research, J.L.M., D.P.I.C., A.R.W., R.L.E., G.P.; clinical studies, J.L.M., D.P.I.C., R.L.E., D.G.M., G.P.; statistical analysis, J.L.M., A.R.W., R.L.E., A.L.B., M.K., G.P.; and manuscript editing, all authors

Disclosures of Conflicts of Interest: J.L.M. disclosed no relevant relationships. D.P.I.C. disclosed no relevant relationships. A.R.W. disclosed no relevant relationships. R.L.E. disclosed no relevant relationships. A.L.B. disclosed no relevant relationships. D.G.M. disclosed no relevant relationships. M.K. Activities related to 
the present article: disclosed no relevant relationships. Activities not related to the present article: is a consultant for Vida Diagnostics. Other relationships: disclosed no relevant relationships. G.P. disclosed no relevant relationships.

\section{References}

1. Vestbo J, Hurd SS, Agustí AG, et al. Global strategy for the diagnosis, management, and prevention of chronic obstructive pulmonary disease: GOLD executive summary. Am J Respir Crit Care Med 2013;187(4):347-365.

2. Nakano Y, Muro S, Sakai H, et al. Computed tomographic measurements of airway dimensions and emphysema in smokers. Correlation with lung function. Am J Respir Crit Care Med 2000;162(3 Pt 1):1102-1108.

3. Gevenois PA, De Vuyst P, de Maertelaer V, et al. Comparison of computed density and microscopic morphometry in pulmonary emphysema. Am J Respir Crit Care Med 1996;154(1):187-192.

4. Galbán CJ, Han MK, Boes JL, et al. Computed tomography-based biomarker provides unique signature for diagnosis of COPD phenotypes and disease progression. Nat Med 2012;18(11):1711-1715.

5. Fernández-Baldera A, Hatt CR, Murray S, et al. Correcting Nonpathological Variation in Longitudinal Parametric Response Maps of CT Scans in COPD Subjects: SPIROMICS. Tomography 2017;3(3):138-145.

6. Belloli EA, Degtiar I, Wang X, et al. Parametric Response Mapping as an Imaging Biomarker in Lung Transplant Recipients. Am J Respir Crit Care Med 2017;195(7):942-952

7. Regan EA, Hokanson JE, Murphy JR, et al. Genetic epidemiology of COPD (COPDGene) study design. COPD 2010;7(1):32-43.

8. Couper D, LaVange LM, Han M, et al. Design of the Subpopulations and Intermediate Outcomes in COPD Study (SPIROMICS). Thorax 2014;69(5): 491-494.

9. Thomashow MA, Shimbo D, Parikh MA, et al. Endothelial microparticles in mild chronic obstructive pulmonary disease and emphysema. The Multi-Ethnic Study of Atherosclerosis Chronic Obstructive Pulmonary Disease study. Am J Respir Crit Care Med 2013;188(1):60-68.

10. Bourbeau J, Tan WC, Benedetti A, et al. Canadian Cohort Obstructive Lung Disease (CanCOLD): Fulfilling the need for longitudinal observational studies in COPD. COPD 2014;11(2):125-132.

11. Vestbo J, Anderson W, Coxson HO, et al. Evaluation of COPD Longitudinally to Identify Predictive Surrogate End-points (ECLIPSE). Eur Respir J 2008;31(4):869-873.

12. Pompe E, Galbán CJ, Ross BD, et al. Parametric response mapping on chest computed tomography associates with clinical and functional parameters in chronic obstructive pulmonary disease. Respir Med 2017;123:48-55.

13. Boes JL, Hoff BA, Bule M, et al. Parametric response mapping monitors temporal changes on lung CT scans in the subpopulations and intermediate outcome measures in COPD Study (SPIROMICS). Acad Radiol 2015;22(2):186-194.

14. Bhatt SP, Soler X, Wang X, et al. Association between Functional Small Airway Disease and FEV1 Decline in Chronic Obstructive Pulmonary Disease. Am J Respir Crit Care Med 2016;194(2):178-184.

15. Virgincar RS, Cleveland ZI, Kaushik SS, et al. Quantitative analysis of hyperpolarized 129Xe ventilation imaging in healthy volunteers and subjects with chronic obstructive pulmonary disease. NMR Biomed 2013;26(4):424-435.

16. Saam BT, Yablonskiy DA, Kodibagkar VD, et al. MR imaging of diffusion of (3) $\mathrm{He}$ gas in healthy and diseased lungs. Magn Reson Med 2000;44(2):174-179.

17. Swift AJ, Wild JM, Fichele S, et al. Emphysematous changes and normal variation in smokers and COPD patients using diffusion 3He MRI. Eur J Radio 2005;54(3):352-358.

18. Kirby M, Pike D, Coxson HO, McCormack DG, Parraga G. Hyperpolarized (3) He ventilation defects used to predict pulmonary exacerbations in mild to moderate chronic obstructive pulmonary disease. Radiology 2014;273(3):887-896.

19. Woods JC, Choong CK, Yablonskiy DA, et al. Hyperpolarized 3He diffusion MRI and histology in pulmonary emphysema. Magn Reson Med 2006;56(6):1293-1300.

20. Kirby M, Pike D, McCormack DG, Lam S, Coxson HO, Parraga G. Longitudinal Computed Tomography and Magnetic Resonance Imaging of COPD: Thoracic Im- aging Network of Canada (TINCan) Study Objectives. Chronic Obstr Pulm Dis 2014;1(2):200-211.

21. Capaldi DP, Zha N, Guo F, et al. Pulmonary Imaging Biomarkers of Gas Trapping and Emphysema in COPD: (3)He MR Imaging and CT Parametric Response Maps. Radiology 2016;279(2):597-608.

22. Kirby M, Owrangi A, Svenningsen S, et al. On the role of abnormal DL(CO) in exsmokers without airflow limitation: symptoms, exercise capacity and hyperpolarised helium-3 MRI. Thorax 2013;68(8):752-759.

23. Pike D, Kirby M, Guo F, McCormack DG, Parraga G. Ventilation heterogeneity in ex-smokers without airflow limitation. Acad Radiol 2015;22(8):1068-1078.

24. Kirby M, Pike D, Sin DD, Coxson HO, McCormack DG, Parraga G. COPD: Do Imaging Measurements of Emphysema and Airway Disease Explain Symptoms and Exercise Capacity? Radiology 2015;277(3):872-880.

25. Pike D, Kirby M, Eddy RL, et al. Regional Heterogeneity of Chronic Obstructive Pulmonary Disease Phenotypes: Pulmonary (3)He Magnetic Resonance Imaging and Computed Tomography. COPD 2016;13(5):601-609.

26. Davis C, Sheikh K, Pike D, et al. Ventilation Heterogeneity in Never-smokers and COPD: Comparison of Pulmonary Functional Magnetic Resonance Imaging with the Poorly Communicating Fraction Derived From Plethysmography. Acad Radiol 2016;23(4):398-405.

27. Miller MR, Hankinson J, Brusasco V, et al. Standardisation of spirometry. Eur Respir J 2005;26(2):319-338.

28. Wanger J, Clausen JL, Coates A, et al. Standardisation of the measurement of lung volumes. Eur Respir J 2005;26(3):511-522.

29. Macintyre N, Crapo RO, Viegi G, et al. Standardisation of the single-breath determination of carbon monoxide uptake in the lung. Eur Respir J 2005;26(4):720735.

30. Jones PW, Quirk FH, Baveystock CM, Littlejohns P. A self-complete measure of health status for chronic airflow limitation. The St. George's Respiratory Questionnaire. Am Rev Respir Dis 1992;145(6):1321-1327.

31. Enright PL. The six-minute walk test. Respir Care 2003;48(8):783-785.

32. Heinrich MP, Jenkinson M, Bhushan M, et al. MIND: modality independent neighbourhood descriptor for multi-modal deformable registration. Med Image Anal 2012;16(7):1423-1435.

33. Paulin GA, Ouriadov A, Lessard E, Sheikh K, McCormack DG, Parraga G. Noninvasive quantification of alveolar morphometry in elderly never- and ex-smokers. Physiol Rep 2015;3(10):e12583.

34. Madani A, Van Muylem A, Gevenois PA. Pulmonary emphysema: effect of lung volume on objective quantification at thin-section CT. Radiology 2010;257(1):260268.

35. Fain SB, Altes TA, Panth SR, et al. Detection of age-dependent changes in healthy adult lungs with diffusion-weighted 3He MRI. Acad Radiol 2005;12(11):13851393.

36. McDonough JE, Yuan R, Suzuki M, et al. Small-airway obstruction and emphysema in chronic obstructive pulmonary disease. N Engl J Med 2011:365(17):1567-1575.

37. Hogg JC, Macklem PT, Thurlbeck WM. Site and nature of airway obstruction in chronic obstructive lung disease. N Engl J Med 1968;278(25):1355-1360.

38. Vasilescu DM, Martinez FJ, Marchetti N, et al. Noninvasive Imaging Biomarker Identifies Small Airway Damage in Severe Chronic Obstructive Pulmonary Disease. Am J Respir Crit Care Med 2019;200(5):575-581.

39. Kaushik SS, Cleveland ZI, Cofer GP, et al. Diffusion-weighted hyperpolarized 129Xe MRI in healthy volunteers and subjects with chronic obstructive pulmonary disease. Magn Reson Med 2011;65(4):1154-1165.

40. Kirby M, Tanabe N, Tan WC, et al. Total Airway Count on Computed Tomography and the Risk of Chronic Obstructive Pulmonary Disease Progression. Findings from a Population-based Study. Am J Respir Crit Care Med 2018;197(1):56-65.

41. Rambod M, Porszasz J, Make BJ, Crapo JD, Casaburi R; COPDGene Investigators. Six-minute walk distance predictors, including CT scan measures, in the COPDGene cohort. Chest 2012;141(4):867-875. 